\title{
TRABAJO COLABORATIVO VIRTUAL COMO ESTRATEGIA ADAPTATIVA EN LA EDUCACIÓN UNIVERSITARIA PERUANA EN TIEMPOS DE PANDEMIA
}

\section{VIRTUAL COLLABORATIVE WORK AS AN ADAPTIVE STRATEGY IN PERUVIAN UNIVERSITY EDUCATION IN TIMES OF PANDEMIC}

\author{
Graciela Martina MONROY CORREA ${ }^{1}$
}

\begin{tabular}{lll} 
Recibido & $:$ & 07.05 .2021 \\
Aprobado & $:$ & 16.11 .2021 \\
Publicado & $:$ & 31.12 .2021 \\
\hline
\end{tabular}

\begin{abstract}
RESUMEN: El objetivo de la investigación fue describir el trabajo colaborativo en entornos virtuales de los estudiantes de la Facultad de Educación de la Universidad Nacional Federico Villarreal. El estudio es de tipo descriptivo y de diseño no experimental, transversal descriptivo. La población estuvo conformada por 303 estudiantes y la muestra por 168 alumnos de la Escuela Profesional de Educación Primaria y Secundaria de la Facultad de Educación. Para el recojo de información se utilizó una escala de medición de tipo Likert de tres niveles. La validez de contenido a través de la V-Aiken arrojó un valor de 0.80 ; la validez de constructo de varianza total, $42.460 \%$, y la confiabilidad a través de Alfa Cronbach, 0.748. Los resultados demuestran que el trabajo colaborativo implementado en entornos virtuales facilita la interrelación entre los estudiantes, permite desarrollar habilidades sociales y favorece el valor de la responsabilidad individual y compartida. Por otro lado, los resultados evidencian que, el 91.1\%, de los estudiantes de la Facultad de Educación siempre realizan trabajo colaborativo, mientras que, un $9 \%$ a veces lo realiza. Asimismo, se concluye que, a mayor edad, mayor disposición a realizar trabajo colaborativo; obteniendo una ligera diferencia de los varones en relación a las mujeres.
\end{abstract}

Palabras clave: Trabajo colaborativo, aprendizaje colaborativo, competencias sociales, interdependencia positiva, habilidades sociales.

\begin{abstract}
The objective of this research was to describe the collaborative work in virtual environments of the students of the Faculty of Education of Universidad Nacional Federico Villarreal. The nature of this study is descriptive and has a non-experimental, cross-sectional descriptive design. The population consisted of 303 students and the sample of 168 students from the Professional School of Primary and Secondary Education of the Faculty of Education. In order to collect the information, a three-level Likert-type measurement scale was used. The content validity through the V-Aiken gave a result of 0.80; the construct validity of total variance, $42.460 \%$, and the reliability through Alpha Cronbach, 0.748. The results showed that collaborative work implemented in virtual environments facilitates interrelation between students, allows the development of social skills and favors the value of individual and shared responsibility. On the other hand, the results showed that $91.1 \%$ of the students of the Faculty of Education always carry out collaborative work, while 9\% sometimes do it. Likewise, it is concluded that the older the age, the greater the willingness to carry out collaborative work, obtaining a slight difference of men in relation to women.
\end{abstract}

Keywords: Collaborative work, Collaborative learning, Social skills, Positive interdependence, Social skills.

\footnotetext{
${ }^{1}$ Magister en Educación, Universidad Nacional Federico Villareal, Lima - Perú. Email: gmonroy@unfv.edu.pe ORCID: https://orcid.org/0000-0002-7908-0968
} 


\section{Journal of the Academy $|128|$}

\section{INTRODUCCIÓN}

La pandemia del COVID - 19 afectó a todos los sectores de la sociedad y el sistema educativo no fue ajeno a ello, a raíz del anuncio de Tedros Adhanom Ghebreyesus, director general de la Organización Mundial de la Salud (OMS), las universidades tuvieron que pasar de clases presenciales a una modalidad virtual, para la cual no estaban preparados los docentes ni los alumnos (Organización Mundial de la Salud, 2021). Como consecuencia, el aprendizaje de los estudiantes fue suspendido repentinamente con el fin de prevenir la propagación de la enfermedad. En el ámbito educativo, el impacto a nivel mundial fue del 60\% y en el Perú 1895907 alumnos vieron interrumpidos sus estudios universitarios (Vivanco-Vidal et al., 2020). Al respecto, la Organización de las Naciones Unidas para la Educación, la Ciencia y la Cultura - UNESCO (2020) sostiene que los recintos universitarios con la finalidad de evitar la expansión de la COVID-19 y dar continuidad al servicio educativo implantaron campus virtuales, medios de comunicación y diversos entornos digitales, así como también reprogramaron los calendarios académicos.

Con la incorporación de clases en entornos virtuales, disminuyó la interacción social y aumentaron las exigencias académicas y tecnológicas. Por lo tanto, se evidenció una brecha digital y generacional que generó en los estudiantes presión, estrés y ansiedad (Sanz, et al., 2020). Esta problemática no fue ajena al Perú, que al inicio de la pandemia contaba solo con el $27 \%$ de universidades autorizadas para brindar programas semipresenciales, de las cuales 23 universidades eran privadas y 2, públicas. Ante ello, el Ministerio de Educación (MINEDU) buscó asegurar la continuidad de estudios en las universidades públicas de todo el país; se promulgó la normatividad para la educación virtual, se brindó asesoría técnica especializada por organismos nacionales e internacionales, se habilitó a las universidades públicas a contratar el servicio de internet y mejorar su conectividad y se otorgaron 36200 becas para traslado y continuidad de estudios. Es así que, en abril, dos universidades públicas iniciaron clases remotas hasta llegar a 51 en el mes de agosto (Mori, 2021).

Por su parte, los maestros tuvieron que capacitarse sobre la marcha en el uso de herramientas digitales y buscar estrategias para desarrollar clases dinámicas y participativas que permitan concretar aprendizajes significativos. Por el lado de los estudiantes, pasaron de aulas presenciales donde podían interactuar con sus pares a estar solos y distantes de sus amigos y compañeros. 


\section{Journal of the Academy $|129|$}

Ante esta realidad, el MINEDU puso a disposición de los docentes universitarios la plataforma Conectados, espacio donde los maestros encuentran diversos tutoriales sobre recursos digitales tales como Socrative, Padlet, ¡Kahoot!, Plickers, EdPuzzle, Classflow, Learning apps, Quizizz, entre otros. Estas herramientas facilitan el desarrollo de clases de manera remota, ya que permiten crear contenido, actividades lúdicas y evaluaciones en línea; además de ser espacios de interacción y colaboración entre los alumnos, quienes de manera colectiva sintetizan y organizan información, plantean alternativas de solución ante problemas reales o simulados (casuísticas) y elaboran informes sobre los proyectos realizados (MINEDU, 2020).

Una de las estrategias que favorece la interacción e interrelación entre los estudiantes es el trabajo colaborativo, al respecto, Revelo-Sánchez et al., (2018) consideran que el trabajo colaborativo es una estrategia didáctica que, a través de la interacción de los participantes y el intercambio de ideas e información genera la construcción de aprendizajes significativos. El MINEDU (2020) plantea que el trabajo colaborativo se puede emplear también en entornos virtuales a través de foros de discusión y blogs, pues estos espacios permiten a los estudiantes agruparse, discutir y debatir sin la necesidad de estar presentes físicamente.

El trabajo colectivo permite a los miembros del equipo expresarse con mayor soltura e intercambiar opiniones sin temor a ser juzgados, al respecto, Villasana y Dorrego (2012) consideran al trabajo colaborativo una metodología que desarrolla habilidades sociales y favorece la conversación, aceptación y oposición asertiva en los estudiantes universitarios, mientras que Cotán et al., (2020) sostienen que el trabajo colaborativo online es una herramienta didáctica que ayuda a la construcción social del conocimiento, donde los estudiantes pueden comparar, reflexionar y sintetizar aspectos que determinen su propio aprendizaje.

\section{MARCO TEÓRICO}

Hoy en día, la sociedad actual demanda de profesionales cada vez más competentes, que sepan afrontar los desafíos y retos de una sociedad en constante cambio, que realicen propuestas creativas e innovadoras, que planteen soluciones a los diversos problemas que se presentan en la organización y que trabajen de forma colaborativa con el fin de obtener mejores resultados y una mejor producción. Ya no se concibe la idea de un trabajo aislado e individual sino todo lo contrario, se apuesta por el trabajo en equipo a través de actividades colaborativas. Al 


\section{Journal of the Academy | $130 \mid$}

respecto, Amherdt et al. (2000), sostienen que en los últimos años, las organizaciones apuestan por desarrollar y promover la cooperación, así como fomentar la participación de los empleados y apostar por líderes asertivos, que promuevan el trabajo en equipo y planteen alternativas de solución consensuadas. Por ello, es importante desarrollar en los futuros profesionales, no solo competencias cognitivas, investigativas y ambientales; sino también competencias sociales, tan importante en este mundo globalizado.

Las competencias sociales se desarrollan en los estudiantes a través de actividades colaborativas que les permiten interactuar con sus pares y compartir la responsabilidad por los resultados obtenidos. Esto se respalda en los propuestos de Piaget, quien argumenta que el conocimiento se adquiere por la incorporación de la nueva información a los esquemas preexistentes, los cuáles se modifican y organizan por asimilación y acomodación mediante la actividad del estudiante. (Saldarriaga-Zambrano et al., 2016).

En esa misma línea, Vigotsky (1979) toma como punto de partida los propuestos de Piaget y plantea la idea de la doble formación, afirmando que la función cognitiva aparece primero en el ámbito social e interpersonal, y posteriormente se reconstruye en el plano individual e intrapersonal; para Vigotsky, todas las funciones superiores se producen a través de las relaciones entre seres humanos (citado en Martínez, 1999).

El ser humano por naturaleza es un ser social y necesita de sus pares para desarrollarse plenamente y esto no es ajeno al campo educativo donde el estudiante construye su propio conocimiento a través de la interacción e interrelación con sus pares y con ayuda del docente que brinda el andamiaje necesario para concretar aprendizajes significativos, es por ello que los estudiantes al trabajar en equipos colaborativos aprenden mucho más que si lo realizara de forma individual.

La construcción social permite intercambiar saberes previos, aclarar dudas y/o desterrar conocimientos que se daban por sentado. En adición, Revelo-Sánchez et al., (2018), sostienen que los estudiantes, al trabajar de forma colaborativa aprenden más de lo aprenderían por sí solos, debido a la interacción entre los integrantes del equipo, quienes establecen diferencias y contrastan sus puntos de vista para construir nuevos conocimientos. A este proceso se le denomina aprendizaje colaborativo. 


\section{Journal of the Academy $|131|$}

Jhonson y Jhonson (1991) fueron los pioneros en sentar las bases de las actividades cooperativas, sostienen que el trabajo colaborativo debe realizarse en grupos pequeños, donde los estudiantes trabajen juntos y aprovechen al máximo el aprendizaje individual y el que se produce en la interrelación (Sánchez y Paniagua, 2005).

\section{Dimensiones del trabajo colaborativo}

- Interdependencia positiva: permite la relación entre todos los miembros del equipo, donde cada uno depende del otro integrante para llegar a la meta.

- Responsabilidad individual: implica el compromiso personal y con todos los integrantes del grupo.

- Interacción: fomenta el desarrollo de habilidades interpersonales, estableciendo estrategias efectivas de aprendizaje.

- Habilidades sociales: son necesarias para el desarrollo de las relaciones personales adecuadas y enriquecedoras, (Mesías y Monroy 2017).

En el 2005, la Organización para la Cooperación y el Desarrollo Económico (OCDE) planteó que el desarrollo sostenible y la cohesión social dependen del desarrollo de competencias que involucren no solo el conocimiento, sino también las destrezas, actitudes y valores. Es así que, en 1997, nace el Proyecto de Definición y Selección de Competencias (DeSeCo), con el fin de crear competencias claves de carácter universal que permitan el desarrollo de la autonomía personal y el trabajo en equipo (Toribio, 2010).

El Proyecto DeSeCo (2005), establece tres de categorías de competencias que todo profesional debe tener: el uso de la tecnología de forma interactiva, la interacción en grupos heterogéneos y actuar de forma autónoma. La segunda competencia promueve la habilidad para relacionarse con otras personas, resolver conflictos y trabajar cooperativamente en el logro de los objetivos (Toribio, 2010).

Como vemos, el trabajo colaborativo favorece el desarrollo de competencias sociales muy necesarias en toda organización. En el ámbito educativo, debido a los buenos resultados obtenidos, muchas universidades vienen aplicando esta estrategia desde hace un buen tiempo. En la actualidad, investigaciones como la de Duran y Parra (2014), sostienen que las habilidades sociales permiten al estudiante universitario tomar conciencia de sí mismo y de su medio ambiente con la finalidad de desempeñar funciones sociales en todos los ámbitos de su 


\section{Journal of the Academy $|132|$}

vida, las cuáles se concretan a través de actividades y procesos socioeducativos. Mora-Vicariol y Hooper-Simpson (2016) plantean que el trabajo colaborativo promueve la organización, el autoaprendizaje y las habilidades de comunicación

Otro punto importante a destacar son los hallazgos de Rodríguez y Espinoza (2017), quienes llegaron a la conclusión de que los estudiantes universitarios entre 18 y 23 años emplean con mayor frecuencia la estrategia del trabajo colaborativo debido a que les permite obtener un óptimo desempeño y un buen aprendizaje autodirigido. Adicionalmente Adriaenséns (2015) afirma que los varones al trabajar colaborativamente perciben mayores beneficios que las mujeres en relación al aprendizaje colaborativo.

El trabajo colaborativo como estrategia que facilita la construcción de comunidades y aprendizajes significativos no solo favorece a los estudiantes universitarios, sino también a los alumnos de educación básica, lo cual está normado en el Currículo Nacional que propone una formación integral con una sólida formación ética, donde los estudiantes estén “... dispuestos a procurar su bienestar y el de los demás trabajando de forma colaborativa, cuidando el ambiente, investigando sobre el mundo que los rodea, siendo capaces de aprender permanentemente, y dotados con iniciativa y emprendimiento...” (MINEDU, 2017, p.13). Por ello, las competencias sociales que se desarrollan a través del trabajo colaborativo son muy necesarias en nuestra sociedad actual, donde cada vez más se busca profesionales que dominen las competencias propias de su profesión, pero que también sean tolerantes, empáticos, organizados, solidarios, creativos y capaces de aceptar opiniones distintas a las propias.

\section{METODOLOGÍA}

El estudio corresponde a una investigación de tipo descriptiva, debido a que se exponen las características, rasgos particulares y tendencias de un grupo determinado, ahondando en las dimensiones del fenómeno (Hernández et al., 2014), mientras que, el diseño es transversal descriptivo, debido a que no se manipulará la variable de estudio (Hernández et. al., 2014). La finalidad de la investigación es describir el nivel del trabajo colaborativo en los estudiantes de pregrado de la Facultad de Educación de la Universidad Nacional Federico Villarreal. 


\section{Población y muestra}

En el presente estudio se consideró a la población de la Escuela Profesional de Educación Primaria (EPEP), conformada por 168 estudiantes y a la Escuela Profesional de Educación Secundaria (EPES), con 135 alumnos de la especialidad de inglés. La población total fue de 303 estudiantes de ambos sexos. Para obtener la muestra se empleó el muestreo probabilístico. Al respecto, Salgado-Lévano (2018) sostiene que todos los participantes que forman parte de la población tienen la misma probabilidad de ser incluidos en la muestra, para hallar la muestra se aplicó la siguiente fórmula:

$$
\mathrm{n}=\frac{\mathrm{Z}^{2} \mathrm{pqN}}{\mathrm{e}^{2}(\mathrm{~N}-1)+\mathrm{Z}^{2} \mathrm{pq}}
$$

La muestra estuvo conformada por 168 estudiantes de pregrado de ambas escuelas, tal como se puede apreciar en la tabla 1.

\section{Tabla 1}

\section{Distribución de la población y muestra}

\begin{tabular}{lccc}
\hline \multicolumn{1}{c}{ IEA } & Población & $\%$ & Muestra \\
\hline $\begin{array}{l}\text { Escuela Profesional de Educación } \\
\text { Primaria }\end{array}$ & 168 & $54.5 \%$ & 92 \\
$\begin{array}{l}\text { Escuela Profesional de Educación } \\
\text { Secundaria, especialidad de Inglés. }\end{array}$ & 135 & $45.5 \%$ & 76 \\
Total & 303 & $100 \%$ & 168 \\
\hline
\end{tabular}

Fuente: Elaboración propia

\section{Instrumento de recolección de datos}

Para el recojo de información se empleó el instrumento Escala de trabajo colaborativo diseñado por Mesías y Monroy (2017), tal como se puede observar en la tabla N 2. 
Tabla 2

Ficha técnica

\begin{tabular}{ll}
\hline & Escala de Medición de trabajo colaborativo \\
\hline Autores & $\begin{array}{l}\text { Mesías Rojas Zulma Zaret y Monroy Correa } \\
\text { Graciela Martina (2017) }\end{array}$ \\
& $\begin{array}{l}\text { Interdependencia positiva } \\
\text { Responsabilidad individual }\end{array}$ \\
Áreas de evaluación & Habilidades sociales \\
& $\begin{array}{l}135 \text { docentes de una red de colegios particulares } \\
\text { de la ciudad de Lima }\end{array}$ \\
Prueba piloto & V de Aiken 0.80 \\
Validez de contenido & Varianza 42.460\% \\
Validez de Constructo & Alfa de Cronbach 0.748 \\
Confiabilidad & 1 Nunca, 2 A veces y 3 Siempre \\
Escala de valoración & Tres \\
Dimensiones & 20 \\
Ítems & 30 minutos \\
Tiempo de aplicación &
\end{tabular}

Fuente: Elaboración propia

\section{Procedimiento}

- Se solicitó el consentimiento informado al Decano de la Facultad de Educación, así como a los estudiantes que conformaron la muestra.

- El instrumento se aplicó en formato digital en un mismo momento.

- Para el procesamiento de información se empleó la hoja de cálculo Excel y el programa estadístico SPSS versión 25.

\section{Resultados}

\section{Descripción de las variables sexo y edad}

Sexo de los participantes

El $81 \%$ de los participantes corresponden al sexo femenino, mientras que el 19\% son de sexo masculino. 


\section{Journal of the Academy $|135|$}

\section{Figura 1}

Variable sexo

Fuente: Elaboración propia

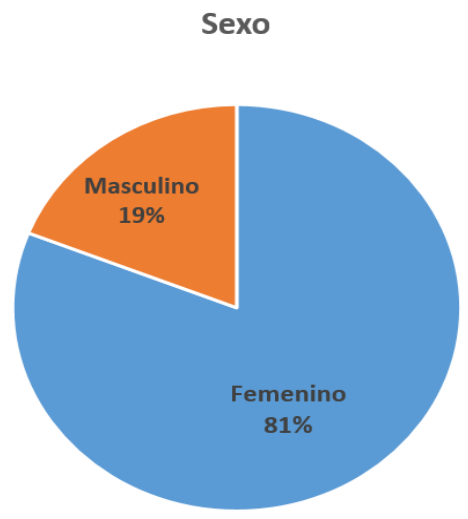

\section{Edad}

En el $16.7 \%$ de los participantes, sus edades fluctúan entre los 18 a 22 años; el $40.5 \%$ entre los 23 a 30 años; mientras que el 42.9\% tienen más de 30 años, como se observa en la tabla 3.

\section{Tabla 3}

\section{Edad de los participantes}

\begin{tabular}{lcc}
\hline \multicolumn{1}{c}{ Sexo } & Frecuencia & Porcentaje \\
\hline Entre $18-22$ & 28 & $16,7 \%$ \\
Entre $23-30$ & 68 & $40,5 \%$ \\
Más de 30 años & 72 & $42,9 \%$ \\
Total & 168 & $100 \%$ \\
\hline
\end{tabular}

Fuente: Elaboración propia

\section{Descripción de las dimensiones del trabajo colaborativo}

\section{Tabla 4}

\section{Dimensión Interdependencia positiva}

\begin{tabular}{lcccc}
\hline Escala & Frecuencia & Porcentaje & $\begin{array}{c}\text { Porcentaje } \\
\text { válido }\end{array}$ & $\begin{array}{c}\text { Porcentaje } \\
\text { acumulado }\end{array}$ \\
\hline A veces & 47 & $28 \%$ & 28 & 28 \\
Siempre & 121 & $72 \%$ & 72 & 100 \\
Total & 168 & $100 \%$ & 100 & \\
\hline
\end{tabular}

Fuente: Elaboración propia 


\section{Journal of the Academy $|136|$}

De acuerdo a la tabla estadística se evidencia que $28 \%$ de estudiantes se ubican en la escala de $a$ veces en relación a la Dimensión Interdependencia positiva, mientras que $72 \%$ se ubican dentro de la escala siempre.

Estos resultados demuestran una adecuada interdependencia positiva entre los alumnos, es decir, cada uno depende del otro para conseguir objetivos comunes, los cuales se logran a través de una interacción constante que anima la colaboración, la comunicación eficaz y el intercambio de recursos y materiales (Lobato, 1997).

\section{Tabla 5}

\section{Dimensión Responsabilidad individual}

\begin{tabular}{lcccc}
\hline Escala & Frecuencia & Porcentaje & $\begin{array}{c}\text { Porcentaje } \\
\text { válido }\end{array}$ & $\begin{array}{c}\text { Porcentaje } \\
\text { acumulado }\end{array}$ \\
\hline A veces & 33 & $19,6 \%$ & 19,6 & 19,6 \\
Siempre & 135 & $80,4 \%$ & 80,4 & 100 \\
Total & 168 & $100 \%$ & 100,0 & \\
\hline
\end{tabular}

Fuente: Elaboración propia

De acuerdo a la tabla estadística se observa que el 19,6\% de estudiantes se ubican en la escala de $a$ veces en relación a la Dimensión Responsabilidades Individual, mientras que $80.4 \%$ alumnos se ubican dentro de la escala siempre.

Los resultados demuestran un alto grado de responsabilidad individual por parte de los estudiantes universitarios al trabajar colaborativamente, ya que no es posible concretar las metas si no existe una responsabilidad individual y a la vez compartida por todos los integrantes del equipo. Al respecto, Traver (2014) sostiene que la responsabilidad individual es un elemento básico del trabajo colaborativo y un aspecto esencial en la formación universitaria, además de ser una importante competencia personal, social y profesional.

\section{Tabla 6}

\section{Dimensión Habilidades sociales}

\begin{tabular}{ccccc}
\hline Escala & Frecuencia & Porcentaje & $\begin{array}{c}\text { Porcentaje } \\
\text { válido }\end{array}$ & $\begin{array}{c}\text { Porcentaje } \\
\text { acumulado }\end{array}$ \\
\hline Nunca & 1 & $6 \%$ & 0.6 & 0.6
\end{tabular}


Journal of the Academy $|137|$

\begin{tabular}{lcccc}
\hline A veces & 61 & $36,3 \%$ & 36,3 & 19,6 \\
Siempre & 106 & $63,1 \%$ & 63,1 & 100 \\
Total & 168 & $100 \%$ & 100,0 & \\
\hline
\end{tabular}

Fuente: Elaboración propia

De acuerdo a la tabla estadística y la figura, se evidencia que el $6 \%$ de estudiantes se ubican en la escala de nunca, mientras que el $36,3 \%$ se encuentran en la escala $A$ veces, y un $63,1 \%$ en la escala siempre, en relación a la dimensión Habilidades sociales.

De los resultados, se deduce que si bien hay un porcentaje considerable de estudiantes que han desarrollado habilidades sociales, también hay un grupo considerable que no las han desarrollado plenamente, y ello se debe a que las habilidades sociales son conductas emitidas en un contexto interpersonal que implica sentimientos, actitudes, deseos, opiniones o derechos que salen a flote ante una situación determinada y que generalmente facilitan la resolución de problemas y reducen las fricciones en los integrantes del equipo (Campo y Martínez, 2009).

Las habilidades sociales, o también llamadas competencias sociales, no se adquieren de un momento a otro, estas se desarrollan a lo largo de la vida y entornos sociales. Por ello, es importante promover desde la primera infancia el trabajo colaborativo como estrategia para facilitar su desarrollo.

\section{Tabla 7}

Trabajo colaborativo según el sexo de los participantes

\begin{tabular}{lccccc}
\hline $\begin{array}{c}\text { Trabajo } \\
\text { colaborativo }\end{array}$ & Femenino & $\%$ & Masculino & $\%$ & Total \\
\hline Nunca & 1 & $1 \%$ & 0 & $0 \%$ & 1 \\
A veces & 59 & $43 \%$ & 12 & $37.5 \%$ & 71 \\
Siempre & 76 & $56 \%$ & 20 & $62.5 \%$ & 96 \\
Total & 136 & $100 \%$ & 32 & $100 \%$ & 168 \\
\hline
\end{tabular}

Fuente: Elaboración propia 


\section{Journal of the Academy $|138|$}

Según los datos obtenidos, se evidencia que el $56 \%$ de personas del sexo femenino realizan siempre trabajo colaborativo, mientras que un $43 \%$ lo realiza a veces y un $1 \%$ nunca lo realiza. Por otro lado, el $63 \%$ de personas del sexo masculino siempre realiza actividades colaborativas mientras que un $38 \%$ lo realiza a veces.

\section{Tabla 8}

Trabajo colaborativo según la edad de los participantes

\section{Edad}

\begin{tabular}{|c|c|c|c|c|c|c|c|}
\hline $\begin{array}{c}\text { Trabajo } \\
\text { colaborativo }\end{array}$ & $\begin{array}{c}\text { entre } 18- \\
22\end{array}$ & $\%$ & $\begin{array}{l}\text { entre } \\
23-30\end{array}$ & $\%$ & más de 30 & $\%$ & Total \\
\hline A veces & 5 & $18 \%$ & 5 & $7 \%$ & 5 & $7 \%$ & 15 \\
\hline Siempre & 23 & $82 \%$ & 63 & $93 \%$ & 67 & $93 \%$ & 153 \\
\hline Total & 28 & & 68 & & 72 & & 168 \\
\hline
\end{tabular}

Fuente: Elaboración propia

De acuerdo a la tabla 8 , se evidencia que el $18 \%$ de los estudiantes cuyas edades fluctúan entre los 18 - 22 años a veces realizan trabajo colaborativo, mientras que el $82 \%$ siempre lo realiza. Por otro lado, el 7\% de alumnos cuyas edades están entre los 23 - 30 años a veces realizan trabajo colaborativo en relación al $93 \%$ que siempre lo hace.

Finalmente, se evidencia que el 7\% de estudiantes que tienen más de 30 años a veces realizan trabajo colaborativo, mientras que $93 \%$ siempre lo realizan.

\section{Tabla 9}

\section{Tabla cruzada por escuelas}

\begin{tabular}{lcccccc}
\hline & & \multicolumn{2}{c}{ Escuelas } & \multicolumn{2}{c}{ Total } \\
& & Primaria & Secundaria & \\
\hline Trabajo & A veces & 9 & $10 \%$ & 6 & $8 \%$ & 15 \\
Colaborativo & & 83 & $90 \%$ & 70 & $92 \%$ & 153 \\
& Siempre & & & & & \\
& Total & 92 & $100 \%$ & 76 & $100 \%$ & 168 \\
\hline
\end{tabular}

Fuente: Elaboración propia

Según la tabla 9, se evidencia que el $10 \%$ de los estudiantes de Escuela Profesional de 
Journal of the Academy | 139 |

Educación Primaria (EPEP) realizan trabajo colaborativo a veces, mientras que, la Escuela Profesional de Educación Secundaria (EPES) obtuvo un 8\% en la misma categoría. Por otro lado, la Escuela Profesional Educación Primaria obtuvo un 90\% en la categoría Siempre en relación al trabajo colaborativo, y la Escuela Profesional de Educación Secundaria alcanzó un valor de $92 \%$.

\section{Tabla 10}

Trabajo colaborativo de los estudiantes de pre grado de la Facultad de Educación.

\begin{tabular}{lcccc}
\hline $\begin{array}{c}\text { Trabajo } \\
\text { Colaborativo }\end{array}$ & Frecuencia & $\%$ & $\begin{array}{c}\text { Porcentaje } \\
\text { válido }\end{array}$ & $\begin{array}{c}\text { Porcentaje } \\
\text { acumulado }\end{array}$ \\
\hline A veces & 15 & $9 \%$ & 8.9 & 8,9 \\
Siempre & 153 & $91 \%$ & 91.1 & 100 \\
Total & 168 & $100 \%$ & 100 & \\
\hline
\end{tabular}

Fuente: Elaboración propia

Según los datos obtenidos se evidencia que el $9 \%$ de los estudiantes de la Facultad de Educación realizan trabajo colaborativo a veces, mientras que el 91\% siempre lo realiza.

De ello, se puede deducir que el trabajo colaborativo es empleado por los alumnos de la Facultad de Educación en un porcentaje alto. El trabajo colaborativo en espacios virtuales ha demostrado efectividad en los resultados y en el logro de los objetivos de aprendizajes, debido a que esta estrategia permite tener una organización claramente definida, conociendo previamente qué se va hacer (metas) cómo se va hacer (procedimientos) y quién lo va hacer (funciones). Permite, además, percibir y evidenciar el esfuerzo individual y colectivo, los aportes, valores, actitudes y el desarrollo de habilidades y competencias de cada uno de los miembros del equipo (Galindo, 2015).

\section{Discusión de resultados}

La presente investigación ha consistido en describir el trabajo colaborativo en entornos virtuales como estrategia de aprendizaje de los estudiantes de pregrado de la Facultad de Educación de la Universidad Nacional Federico Villarreal. Al respecto, se encontraron indicadores altos en las dimensiones Interdependencia positiva y Responsabilidad social, con $72 \%$ y $80.4 \%$ respectivamente; mientras que, la dimensión Habilidades sociales obtuvo un valor de 63,1\%, ligeramente inferior a las dos anteriores, lo que demuestra que todavía hay un número 


\section{Journal of the Academy $|140|$}

considerable de estudiantes que tiene dificultad para interactuar con sus pares y expresar sus ideas libremente. Ello podría responder a que las habilidades sociales se adquieren paulatinamente a través de la sociabilización con el entorno, sin embargo, estas habilidades son necesarias para trabajar en equipos colaborativos ya que facilitan la escucha activa, la empatía y tolerancia entre todos los miembros del equipo, y por ende, la construcción de aprendizajes más significativos.

Por otro lado, en cuanto al sexo de los participantes y su relación con el trabajo colaborativo, los hallazgos permiten afirmar que son las personas de sexo masculino quienes desarrollan mayor trabajo colaborativo en relación a las mujeres.

En cuanto a la edad de los participantes y el trabajo colaborativo, se puede afirmar que a mayor edad (23 años hacia adelante), los estudiantes universitarios desarrollan mayor trabajo colaborativo.

El trabajo colaborativo en entornos virtuales es aceptado por gran parte de los estudiantes de la Facultad de Educación de la Universidad Nacional Federico Villarreal, debido a los múltiples beneficios que obtienen al trabajar en equipos colaborativos. El trabajo colectivo facilita construcción de comunidades de aprendizaje, el desarrollo de habilidades sociales, la responsabilidad compartida y la interacción entre compañeros tan venida a menos debido al aislamiento social como consecuencia de la COVID-19.

La estrategia del trabajo colaborativo se puede aplicar en cualquier profesión, por ello, es importante que los docentes universitarios apliquen esta estrategia de aprendizaje de forma continua, y más aún los docentes que enseñan en la Facultad de Educación y que forman a los futuros profesores de educación básica, pues, ellos también aplicarán esta estrategia con sus alumnos, la cual está normada desde el año 2016 en el Currículo Nacional.

\section{CONCLUSIONES}

Los resultados de la investigación permiten determinar que el trabajo colaborativo como estrategia de aprendizaje en la educación remota fue bien aceptado por los estudiantes de la Facultad de Educación, encontrando mayor disposición por parte de los varones en relación a las damas, por otro lado, el trabajo colaborativo permitió a los estudiantes interactuar con sus pares, dialogar, investigar, sacar conclusiones, plantear soluciones y concretar los proyectos 


\section{Journal of the Academy | 141 |}

que se trabajan en las diversas asignaturas que forman parte de su formación profesional. Este espacio colectivo, permitió que trabajen juntos, aunque físicamente distantes, pero, aun así, es más motivador que participar en una clase sincrónica unidireccional.

Por ello, es importante que las universidades promuevan el trabajo colaborativo docente como una práctica permanente, que permita formar comunidades de aprendizaje, donde los maestros puedan intercambiar recursos, contenido, estrategias y metodologías con sus pares, por otro lado, estos espacios colectivos facilitan el diálogo entre los maestros, sobre sus aciertos y dificultades, además, permiten plantear en equipo alternativas de solución relacionados a su práctica diaria, para brindar aprendizajes significativos y generar en el colectivo una cultura de calidad.

Por su parte el Estado debe incrementar el presupuesto de las universidades públicas, con la finalidad de que estas implementen laboratorios de cómputo, amplíen la banda de internet y continúen capacitando a los docentes sobre el uso de las TIC, ya que cada vez salen más y mejores herramientas. Finalmente, se puede concluir que la pandemia de la COVID-19 nos ha dejado también experiencias positivas en el campo educativo, entre ellas, las nuevas modalidades de estudio en el nivel universitario: presencial admite $20 \%$ de entornos virtuales de aprendizaje; semipresencial admite más del 20 y hasta $70 \%$ de clases en esta modalidad; y a distancia, más del 70\% de clases en entornos virtuales (SUNEDU, 2020). Como vemos, estás modalidades permiten que los estudiantes que geográficamente se encuentran alejados puedan también acceder a una educación universitaria. Otro aspecto positivo a destacar son las competencias digitales adquiridas por los docentes y estudiantes, sin embargo, para no perder lo aprendido, se deben continuar aplicando en la nueva normalidad, y así evitar ser sorprendidos nuevamente por alguna pandemia o por algún evento natural.

\section{RECOMENDACIONES}

- Los docentes deben implementar la estrategia de trabajo colaborativo en todas las asignaturas e incluir herramientas digitales que permitan desarrollar actividades colectivas.

- Aplicar encuestas a los estudiantes para identificar las ventajas y desventajas de trabajar colaborativamente en entornos virtuales.

- Realizar talleres que ayuden a desarrollar habilidades sociales en los estudiantes y así 


\section{Journal of the Academy $|142|$}

obtener una mejor relación con sus pares al realizar actividades colaborativas.

- La modalidad virtual permite desarrollar diversas actividades colectivas, pero es importante que los maestros también monitoreen y brinden retroalimentación oportuna con la finalidad de ayudar a los estudiantes a construir su propio conocimiento.

\section{REFERENCIAS}

Adriaenséns (2015) Aprendizaje Colaborativo en estudios universitarios de grado: medición de su desarrollo en alumnos próximos a graduarse [Tesis de doctorado, Universidad de Deusto]. https://dialnet.unirioja.es/servlet/tesis?codigo=175796

Amherdt, Ch-H., Dupuich-Rabasse F., y Yves D.,(2000) Competences collectives dand les organisations. Emergence, gestion et developpement. Laval. Les Presses Universitarires de Laval.

Campo, L. y Martinez, Y. (2009) Habilidades Sociales en estudiantes de psicología de una universidad privada de la Costa Caribe Colombiana. Revista Iberoamericana e psicología: Ciencia y Tecnología 2(1), 39-59. https://dialnet.unirioja.es/servlet/articulo?codigo=4905122

Cotán, A., Martínez, V., García, I., Gil-Mediavilla, M., y Gallardo-López, J. (2020). El trabajo colaborativo online como herramienta didáctica en Espacios de Enseñanza Superior (EEES). Percepciones de los estudiantes de los Grados en Educación Infantil y Primaria. Revista d'Innovació Docent Universitària, 82-94. https://dialnet.unirioja.es/servlet/articulo?codigo=7256652

Duran, S., y Parra, M. (2014). Diversidad Cultural para promover el desarrollo de habilidades sociales en educación superior. Revista Cultura Educación y Sociedad, 5(1). https://revistascientificas.cuc.edu.co/culturaeducacionysociedad/article/view/995

Galindo, L. (2015) El aprendizaje colaborativo en ambientes virtuales. Cenid. https://dialnet.unirioja.es/servlet/libro?codigo $=652184$

Hernández, R., Fernández, C., y Baptista P. (2014). Metodología de la Investigación. Mc Graw Hill Education.

Johnson D. y Johnson R. (1991). El aprendizaje colaborativo en el aula. Editorial Paidós

Lobato, C. (1997). Hacia una comprensión del aprendizaje cooperativo. Revista de Psicodidáctica, (4), 59-76.

https://www.redalyc.org/pdf/175/17517797004.pdf

Martínez, M. (1999). El enfoque sociocultural en el estudio del desarrollo y la educación. Revista electrónica de investigación educativa, 1 (1). http://redie.uabc.mx/vol1nol/contenidomtzrod.html

Mesías y Monroy (2017) Diseño de un instrumento de medición del trabajo colaborativo en docentes de una institución educativa privada [Tesis de Maestría, Universidad Marcelino Champagnat, Lima, Perú]. https://repositorio.umch.edu.pe/handle/UMCH/317

Ministerio de Educación, (2017). Currículo Nacional de la Educación Básica.

http://www.minedu.gob.pe/curriculo/pdf/curriculo-nacional-de-la-educacion-basica.pdf

Ministerio de Educación (2020). Herramientas TIC para la evaluación de aprendizajes. https://www.minedu.gob.pe/conectados/docentes-recomendaciones.php

Mora-Vicariol, F., y Hooper-Simpson, C. (2016). Trabajo colaborativo en ambientes virtuales de aprendizaje: Algunas reflexiones y perspectivas estudiantiles. Revista Electrónica Educare, 20(2), 1. https://doi.org/10.15359/ree.20-2.19

Mori, J. (2021) I Congreso Conectados. Tendencias educativas: hacia el futuro digital de la educación universitaria. https://congresoconectados.com/

Organización Mundial de la Salud (2021) Cronología de la respuesta de la OMS a la COVID-19. https://www.who.int/es/news/item/29-06-2020-covidtimeline 


\section{Journal of the Academy $|143|$}

Revelo-Sánchez, O., Collazos-Ordóñez, C., y Jiménez-Toledo, J. (2018). El trabajo colaborativo como estrategia didáctica para la enseñanza/aprendizaje de la programación: Una revisión sistemática de literatura. Revista TecnoLógicas, 21(41), 115-134. https://doi.org/10.22430/22565337.731

Rodríguez, R. y Espinoza, L. (2017). Trabajo colaborativo y estrategias de aprendizaje en entornos virtuales en jóvenes universitarios. RIDE Revista Iberoamericana para la Investigación y el Desarrollo Educativo, 7(14) https://www.redalyc.org/articulo.oa?id=498153999006

Salgado-Lévano (2018) Manual de Investigación Teoría y Práctica para hacer la tesis según la metodología cuantitativa. Fondo editorial de la Universidad Marcelino Champagnat.

Sánchez, A., y Paniagua. E., (2005). Construcción de una comunidad virtual de aprendizaje. Pirámide.

Sanz, J., Sainz, J., y Capilla, A. (2020) Efectos de la crisis del coronavirus en la educación.

https://www.flacsi.net/wp-content/uploads/2020/04/EFECTOS-DE-LA-CRISIS-DELCORONAVIRUS-EN-EDUCACI\%C3\%93N.pdf

SUNEDU (2020). Resolución del Consejo Directivo $\mathrm{N}^{\circ}$ 105-2020-SUNEDU/CD (EM) https://www.sunedu.gob.pe/resolucion-del-consejo-directivo-no-105-2020-sunedu-cd-em/

Toribio, L. (2010). Las Competencias Básicas: El nuevo paradigma curricular en Europa. Foro de Educación, 8(12),25-44. https://www.redalyc.org/articulo.oa?id=447544587003

Traver, J. (2014). La responsabilidad individual en el aprendizaje cooperativo: experiencia y reflexiones. Actas de la XX JENUI. 277-284 Universitat Jaume. https://upcommons.upc.edu/bitstream/handle/2099/15475/P277tr lare.pdf

Tünnermann, Carlos. (2011). El constructivismo y el aprendizaje de los estudiantes. Universidades, (48),21-32. ISSN:0041-8935.

https://www.redalyc.org/articulo.oa? id=37319199005

Saldarriaga-Zambrano, P., Bravo-Cedeño, G. y Loor-Rivadeneira, M. (2016). La teoría constructivista de Jean Piaget y su significación para la pedagogía contemporánea. Revista $\begin{array}{llllll}\text { Científica dominio de las ciencias } & \text { (2) }\end{array}$ https://dominiodelasciencias.com/ojs/index.php/es/article/viewFile/298/355

UNESCO (2020) Covid-19 y Educación Superior: Políticas Públicas Nacionales. https://www.iesalc.unesco.org/2020/04/08/covid-19-y-educacion-superior-politicas-publicasnacionales/

Villasana, N., y Dorrego, E. (2012). Habilidades sociales en entornos virtuales de trabajo colaborativo. RIED. Revista Iberoamericana de Educación a Distancia, 10(2). https://doi.org/10.5944/ried.2.10.993

Vivanco-Vidal, A., Saroli-Araníbar, D., Caycho-Rodríguez, T., Carbajal-León, C., y Noé-Grijalva, M. (2020). Ansiedad por Covid-19 y salud mental en estudiantes universitarios. Revista de Investigación en Psicología, 23(2), 197-215. https://doi.org/10.15381/rinvp.v23i2.19241 